\title{
Registering Histological and MR Images of Prostate for Image-Based Cancer Detection
}

\author{
Yiqiang Zhan ${ }^{1,3}$, Michael Feldman ${ }^{2}$, John Tomaszeweski², \\ Christos Davatzikos ${ }^{1}$, and Dinggang Shen ${ }^{1}$ \\ ${ }^{1}$ Sect. of Biomedical Image Analysis, University of Pennsylvania, Philadelphia, PA \\ ${ }^{2}$ Dept. of Pathology and Laboratory Medicine, University of Pennsylvania, Philadelphia, PA \\ ${ }^{3}$ Dept. of Computer Science, Johns Hopkins University, Baltimore, MD \\ yzhan@es.jhu.edu
}

\begin{abstract}
This paper presents a deformable registration method to co-register histological images with MR images of the same prostate. By considering various distortion and cutting artifacts in histological images and also fundamentally different nature of histological and MR images, our registration method is thus guided by two types of landmark points that can be reliably detected in both histological and MR images, i.e., prostate boundary points, and internal salient points that can be identified by a scale-space analysis method. The similarity between these automatically detected landmarks in histological and MR images are defined by geometric features and normalized mutual information, respectively. By optimizing a function, which integrates the similarities between landmarks with spatial constraints, the correspondences between the landmarks as well as the deformable transformation between histological and MR images can be simultaneously obtained. The performance of our proposed registration algorithm has been evaluated by various designed experiments. This work is part of a larger effort to develop statistical atlases of prostate cancer using both imaging and histological information, and to use these atlases for optimal biopsy and therapy planning.
\end{abstract}

\section{Introduction}

With an aging population, prostate cancer ( $\mathrm{PCa}$ ) has become a major medical and socioeconomic problem. Since PCa is relatively less aggressive and is curable if it is detected in the early stage, the early diagnosis of PCa becomes significant for the treatment of this disease. Currently, the common diagnosis method is the transrectal ultrasound (TRUS) guided needle biopsy, following an abnormal digital rectal exam (DRE) or an increasing prostate specific antigen (PSA) level. However, the most widely used biopsy strategy, which randomly picks tissue sample from each of roughly partitioned prostate regions, is more like a blind targeting, resulting in high false negative detection rate. Considering that the $\mathrm{PCa}$ is not uniformly distributed within the prostate capsule, we have developed an optimal needle biopsy strategy for prostate cancer detection, using population-based prostate cancer distribution information obtained from a large cohort of structure-labeled and cancer-identified histological samples of prostates normalized in the standard space [1]. We also developed a texturebased deformable model to segment prostate from TRUS images [2], for warping the optimal needle biopsy locations defined in the standard space to the patient's space. In 
order to achieve a patient-specific optimal needle biopsy, we need to include patientspecific cancer information, which can be obtained from the MR images of the patient by an image-based tissue characterization method $[3,4]$ that have been trained by a sufficient number of prostate MR images with cancerous regions identified.

To identify the cancerous tissues from MR images using a machine learning method, the ground-truth cancer information that is available only in histological images should be mapped to the space of MR images. Currently, the ground-truth cancer information in histological images is manually mapped into MR images by a human expert, i.e., manually labeling the cancerous regions in MR images according to the cancer locations in the corresponding histological images [4]. However, this process is generally very tedious and time-consuming, particularly for a large number of prostate samples required to train a tissue characterization method. Therefore, in this study, we propose an automatic method to co-register histological image with MR image of the same prostate, by mimicking the way that the human experts used to manually align histological and MR images.

It is worth noting that, although multi-modality image registration methods have been extensively investigated, the studies dealing with the registration of histological images with MR images are very limited, probably due to the more complicated and diverse nature of histological images. The recently proposed histological image registration methods could be categorized into two classes. The first class of methods focuses on registering the extracted contours of anatomical structures [5,6]. Although organ contours can be perfectly aligned in these methods, it is not guaranteed that internal structures are also accurately registered. The second class of methods focuses on registering images by maximizing the overall similarity of two images, such as using mutual information $[7,8]$. However, these methods might be misled by various distortions and cutting artifacts in the histological images, since patches with low structural content often lead to morphologically inconsistent local registrations with a low MI response [15].

Considering these limitations, our registration method is based on the common features that can be reliably identified in both histological and MR images, i.e., two types of automatically identified landmarks locating on prostate boundaries and salient internal anatomical regions, which are simply called boundary landmarks and internal landmarks, respectively. In particular, by detecting the internal landmarks commonly available in both histological and MR images, the registration of anatomical structures inside the prostate can be successfully completed, and the mis-registration due to distortion and cutting artifacts can be potentially avoided as the local patches around these landmarks have salient structure information for correspondence matching. The correspondences between landmarks as well as the transformation between histological and MR images can be simultaneously obtained by maximizing an overall similarity function, which integrates the similarities between estimated corresponding landmarks, along with spatial constraints.

\section{Methods}

The identification of meaningful landmarks commonly-available in both histological and MR images is significant for the success of our registration method. Also, the definition for the similarity of the two same-type landmarks respectively in histological and MR images is equally important. These methods are explored in Sections 2.1 and 
2.2 for boundary landmarks and internal landmarks, respectively. With the respective landmarks automatically identified in histological and MR images, their correspondences as well as the transformation between histological and MR images are then established by maximizing an overall similarity function described in Section 2.3.

\subsection{Boundary Landmarks}

Landmarks detection. Since the organ boundaries are usually important for guiding the registration of medical images, the points locating on the prostate boundaries are selected as the first type of landmarks to be used for helping register histological and MR images. In our study, the prostate capsules are first segmented from histological and MR images, respectively, by a manual delineation or an automated segmentation algorithm [2]. Then, a triangular mesh surface is generated for each prostate boundary using a marching cubes algorithm, with the vertices of the surface selected as the boundary landmarks.

Similarity definition. As each boundary landmark is actually a vertex of the surface, its spatial relations with vertices in the neighborhood can be used to describe the geometric features of the boundary landmark. In particular, an affine-invariant attribute vector [10] is used to characterize the geometric anatomy around each boundary landmark. Assuming $x_{i}$ is a boundary landmark under study, its geometric attribute is defined as the volume of the tetrahedron formed by $x_{i}$ and its neighboring vertices. The volumes calculated from vertices in different neighborhood layers are stacked into an attribute vector $F\left(x_{i}\right)$, which characterizes the geometric features of $x_{i}$ from a local to a global fashion. $F\left(x_{i}\right)$ can be further made affine-invariant as $\bar{F}\left(x_{i}\right)$, by normalizing it across the whole surface [10]. By using this attribute vector, the similarity between two boundary landmarks $x_{i}$ and $y_{i}$, respectively in histological and MR images, can be defined by an Euclidean distance between their normalized attribute vectors [10], i.e.,

$$
S\left(x_{i}, y_{j}\right)=1-\left\|\bar{F}\left(x_{i}\right)-\bar{F}\left(y_{j}\right)\right\|
$$

\subsection{Internal Landmarks}

Compared to the boundary landmarks, it is relatively difficult to define the landmarks within the prostate capsules. Inspired by the fact that radiologists usually register histological and MR images by matching the internal blob-like structures, i.e., gland tissues containing fluid, we use those blob-like structures as the second type of landmarks, i.e., internal landmarks, to guide the image registration. However, due to the enlargement or shrinkage of gland tissues during the cutting procedure, the size of the same blob can be different in histological and MR images. Since local structures only exist as meaningful entities over a certain range of scale [11], various blobs can only be detected as salient local structures at their appropriate scales. Also, as we will mention in the next, the similarity between two internal landmarks respectively in histological and MR images is measured by the mutual information between the respective local regions around the two landmarks. Accordingly, the detected size of blob is important information to determine the size of local region to be extracted around each internal landmark, for measuring the local image similarity. 
Landmarks detection. According to the scale-space analysis method [11], the detection of the location and size of blob is completed by three steps. First, a scale-space representation is constructed for a 3 -dimensional image $f(x, y, z)$, i.e.,

$$
L(x, y, z ; s)=g(x, y, z ; s) * f(x, y, z)
$$

where $g(x, y, z ; s)$ is a Gaussian kernel, i.e., $g(x, y, z ; s)=\frac{1}{\left(2 \pi 2^{2}\right)^{3 / 2}} e^{-\left(x^{2}+y^{2}+z^{2}\right) / 2 s^{2}}$. Second, the Laplacian of this scale-space function $L(x, y, z ; s)$ is calculated. For scale-invariant comparison of Laplacian values, we use a normalized Laplacian [11] defined as $\nabla_{n o r m}^{2} L=s^{2} \nabla^{2} L=s^{2}\left(L_{x x}+L_{y y}+L_{z z}\right)$. Finally, the local peak responses over scale space can be scale-invariantly detected as the candidates of interesting local structures, with the corresponding scales indicating the sizes of those local structures.

Since an ideal blob can be modeled as a Gaussian function, its actual location and size can be directly estimated from the scale-space analysis results. For example, if we detect a peak at a location $\left(x_{0}, y_{0}, z_{0}\right)$ with scale $s_{0}$, it indicates that there might be a blob centered at $\left(x_{0}, y_{0}, z_{0}\right)$ with size of $\sqrt{\frac{3}{2}} s_{0}$, which can be mathematically derived next.

Assume an ideal blob locates at $\left(x_{0}, y_{0}, z_{0}\right)$ with the size $\sqrt{\frac{3}{2}} s_{0}$, i.e.,

$$
f(x, y, z)=\frac{A}{\left(\frac{3}{2} s_{0}^{2}\right)^{3 / 2}} e^{-\left(\left(x-x_{0}\right)^{2}+\left(y-y_{0}\right)^{2}+\left(z-z_{0}\right)^{2}\right) / 2\left(\frac{3}{2} s_{0}^{2}\right)}
$$

The scale-space representation of this blob $f$ can be obtained as

$$
L(x, y, z ; s)=\frac{A}{\left(\frac{3}{2} s_{0}^{2}+s^{2}\right)^{3 / 2}} e^{-\left(\left(x-x_{0}\right)^{2}+\left(y-y_{0}\right)^{2}+\left(z-z_{0}\right)^{2}\right) / 2\left(\frac{3}{2} s_{0}^{2}+s^{2}\right)}
$$

At any scale $s$, the normalized Laplacian of $L(x, y, z ; s)$ reaches its maximum at location $\left(x_{0}, y_{0}, z_{0}\right)$, i.e.,

$$
Q(s)=\max _{(x, y, z)} \mid\left(\nabla_{\text {norm }}^{2} L(x, y, z ; s) \mid=\frac{3 A s^{2}}{\left(\frac{3}{2} s_{0}^{2}+s^{2}\right)^{5 / 2}}\right.
$$

The maximum of $Q(s)$ across scales is calculated by differentiating $Q(s)$ with respect to $s$,

$$
\frac{d Q}{d s}=\frac{9 A s\left(s_{0}^{2}-s^{2}\right)}{\left(\frac{3}{2} s_{0}^{2}+s^{2}\right)^{7 / 2}}
$$

As equation (6) equals to zero while $s=s_{0}$, the normalized Laplacian achieves its maximum at $\left(x_{0}, y_{0}, z_{0} ; s_{0}\right)$ in the scale-space, which indicates a blob detected with center $\left(x_{0}, y_{0}, z_{0}\right)$ and size $\sqrt{\frac{3}{2}} s_{0}$.

In summary, by finding the local maxima in a normalized Laplacian map, the centers of blob-like structures are detected and used as candidates for internal landmarks. The expected internal landmarks are further determined as follows. First, the local maxima in the normalized Laplacian map are thresholded, to ensure the detection of salient blob-like structures. Second, average intensity within each detected blob-like structure is computed, to select the bright blobs that we are interested in. Third, the 
extremely-flat blobs are discarded, to avoid the selection of blobs on the boundaries of prostate capsule. Fig 2(a) gives an example of detected internal landmarks, along with their corresponding scales represented by the sizes of circles.

Similarity definition. Once the internal landmarks are determined in both histological and MR images, the normalized mutual information (NMI) [12] is used to evaluate the similarities of two internal landmarks respectively in the histological and MR images. The evaluation of NMI differs from the traditional MI-based registration in the following four aspects. First, NMI is calculated only on the subvolumes around two internal landmarks under comparison. Second, since the sizes of corresponding blobs can be different in histological and MR images due to distortion and cutting artifact, the subvolume around each internal landmark is extracted according to its detected scale, indicating the invariance of our method to scales. The two subvolumes under comparison might have different sizes, but they are normalized to have same size before comparison. Third, in order to capture rich image information around each internal landmark for determining its corresponding landmarks in other modality image, NMI calculated from multiple subvolumes with different sizes around landmarks are integrated. Finally, the subvolumes under comparison are allowed to rotate within a small range of angle, to search for the maximal NMI.

Accordingly, assume two internal landmarks $u$ and $v$ have scales $s_{u}$ and $s_{v}$, respectively, their similarity can be defined as follows.

$$
M(u, v)=\max _{-\alpha \leq \Delta \leq \alpha} \sum_{i=1}^{N} N M I\left\{V\left(u, i \cdot s_{u}\right), T\left(V\left(v, i \cdot s_{v}\right) ; \frac{s_{u}}{s_{v}}, \Delta \theta\right)\right\}
$$

where $V(u, R)$ denotes a spherical subvolume around the landmark $u$ with the radius $R . T(V ; s, \Delta \theta)$ is the transformation operator with a scaling factor $s$ and a rotation factor $\Delta \theta$. The variable $i$ represents different size factor of subvolume to be extracted, and $N$ is the total number of multiple subvolumes used, i.e., $N=3$ in our study. $N M I\{.$, denotes the normalized mutual information between two same-sized spherical volume images.

\subsection{Overall Similarity Function}

After defining the similarity between same-type landmarks, the correspondences between landmarks and the transformation between histological and MR images can be simultaneously estimated, by maximizing an overall similarity function defined next. Assuming the automatically detected boundary landmarks and internal landmarks are $\left\{x_{i} \mid i=1 \cdots I\right\}$ and $\left\{u_{j} \mid j=1 \cdots J\right\}$ in MR image, and $\left\{y_{k} \mid k=1 \cdots K\right\}$ and $\left\{v_{l} \mid l=1 \cdots L\right\}$ in histological image. The overall similarity function can be defined as:

$$
\begin{aligned}
\max _{A, B, h} E(A, B, h)=\max _{A, B, h}\left\{\sum_{i=1}^{I} \sum_{k=1}^{K} a_{i k} S\left(x_{i}, y_{k}\right)+\sum_{j=1}^{J} \sum_{l=1}^{L} b_{j l} M\left(u_{j}, v_{l}\right)\right. \\
\left.-\lambda\left(\sum_{i=1}^{I} \sum_{k=1}^{K} a_{i k} D\left(x_{i}, h\left(y_{k}\right)\right)+\sum_{j=1}^{J} \sum_{l=1}^{L} b_{j l} D\left(u_{j}, h\left(v_{l}\right)\right)+\|W(h)\|^{2}\right)\right\}
\end{aligned}
$$


Here, $h$ is a transformation function that maps histological image to MR image. $A=\left\{a_{i j}\right\}$ and $B=\left\{b_{k l}\right\}$ are the binary correspondence matrix, which subject to $\left\{\sum_{i=1}^{I+1} a_{i k}=1, \sum_{k=1}^{K+1} a_{i k}=1, a_{i k}=\{0,1\}\right\}$ and $\left\{\sum_{j=1}^{J+1} b_{j l}=1, \sum_{l=1}^{L+1} b_{j l}=1, b_{j l}=\{0,1\}\right\}$. The binary correspondence matrix describes the correspondences between landmarks. Note that an extra row and an extra column are added to the binary correspondence matrix for handling the outliers. If a landmark cannot find its correspondence, it is regarded as an outlier and the extra entry of this landmark will be set as $1 . S(\cdot$,$) and M(\cdot$,$) are$ respectively the similarity between boundary landmarks and the similarity between internal landmarks, as defined in equations (1) and (7). $D(\cdot, \cdot)$ denotes the Euclidean distance between two points. $\|W(h)\|^{2}$ is a smoothing term of $h$. According to different smoothing criterion, $h$ can be represented by kernels [14] or thin plate spline [9]. By maximizing the overall similarity function, the correspondences between landmarks and the transformation between histological and MR images can be simultaneously estimated. The correspondences established between internal landmarks after maximizing the overall similarity function are shown in Fig 1 (b).
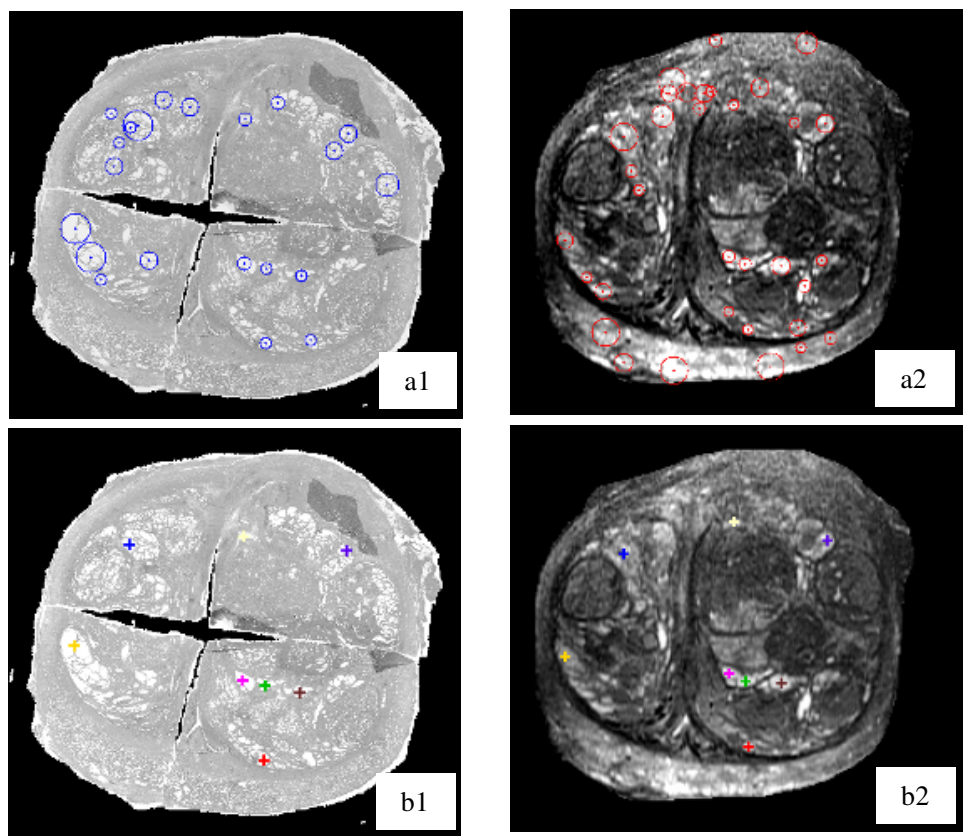

Fig. 1. Detection of internal landmarks and their correspondences. The internal landmarks are detected from histological (a1) and MR images (a2), respectively, with the sizes of circles indicating salient scales. The corresponding landmarks are detected in histological (b1) and MR images (b2). Crosses with the same color denote the corresponding landmarks detected. 
It is worth noting that, in our overall similarity function, the similarity of two landmarks is measured not only by their spatial relations, but also by their geometric and image features. The importance of incorporating image similarity for correspondence detection has also been demonstrated in [14], when registering diffusion tensor images of individual brains.

\section{Experiments}

Histological images and T2-weighted MR of five prostates are used for validating the performance of our registration algorithm. For these images, the corresponding anatomical landmarks and cancerous regions have been manually labeled by an expert. By registering the histological and MR images using a registration algorithm, the correspondences can be automatically established for any points in the histological and MR images, including the manually denoted landmarks. Also, the cancerous regions in the histological images can be automatically warped to the MR images space, therefore automatically labeling the cancerous regions in MR images. In this way, we can compare the difference between the manually-labeled corresponding landmarks and the algorithm-labeled corresponding landmarks. We can also compute the overlay percentage of manually labeled cancerous regions with automatically labeled cancerous regions in MR images.

The performances of three different registration methods are compared. The three registration methods are: (1) Method 1, which is an affine registration algorithm, called FLIRT [13], using mutual information; (2) Method 2, which only uses boundary landmarks to guide the registration; (3) Method 3, i.e., our proposed method. Fig 2 visually demonstrates the performance of three different registration algorithms in warping histological images to match with MR images, which indicates that our method produced the best results in establishing correspondences for landmarks and
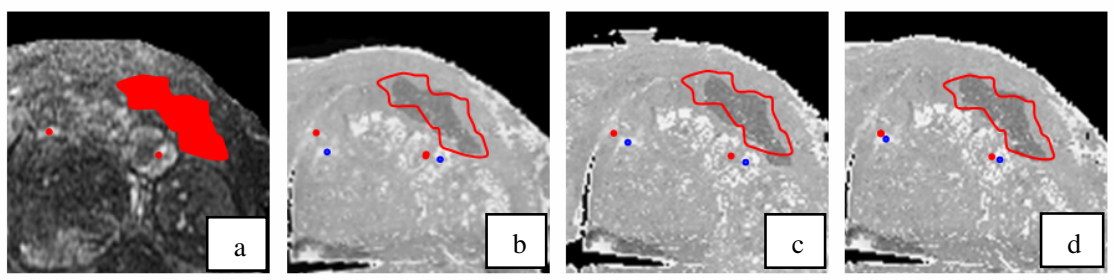

Fig. 2. Comparison of warping histological images to match with MR images by three different registration methods. Two red points and a red region in (a) denote the manually labeled landmarks and cancerous region in an MR image, respectively. For comparison, those red points and the boundary of cancerous region are repeatedly displayed in three warped histological images (b-d) by three registration methods, i.e., Methods 1, 2, and 3, respectively. The blue points in each of three warped histological images (b-d) are the warped landmarks manually labeled in original histological image, as correspondences to those red landmarks in MR image. The dark region in each warped histological image denotes the warped version of the manually labeled cancerous region in the histological image. 
Table 1. Average distances between manually- and automatically-labeled landmarks

\begin{tabular}{cccc}
\hline & Method 1 $(\mathbf{m m})$ & Method 2 $(\mathbf{m m})$ & Method 3 $(\mathbf{m m})$ \\
\hline Subject 1 & 1.31 & 1.03 & 0.77 \\
Subject 2 & 1.81 & 1.05 & 0.97 \\
Subject 3 & 1.25 & 0.97 & 0.76 \\
Subject 4 & 1.43 & 1.09 & 0.81 \\
Subject 5 & 1.53 & 1.03 & 0.87 \\
Mean & 1.47 & 1.03 & 0.82
\end{tabular}

Table 2. Overlay percentage between manually- and automatically-labeled cancerous regions

\begin{tabular}{cccc}
\hline & Method 1 & Method 2 & Method 3 \\
\hline Max & $82.9 \%$ & $87.5 \%$ & $88.3 \%$ \\
Min & $55.9 \%$ & $60.4 \%$ & $64.1 \%$ \\
Average & $71.6 \%$ & $75.5 \%$ & $79.1 \%$
\end{tabular}

labeling cancerous region. The quantitative comparisons on 5 prostate subjects are summarized in Tables 1 and 2, indicating again that our method performs the best among all of three registration methods. We are now planning a large-scale validation study on our method.

\section{Conclusion}

A novel method for co-registeration of histological and MR images of prostate has been proposed in this paper. Instead of matching only the prostate boundaries or evaluating the similarity of intensities in the entire images, our method uses the automatically detected boundary landmarks and internal landmarks to guide the deformable registration of histological and MR images, therefore offering the robustness to various distortions and cutting artifacts in histological images. In particular, the boundary landmarks are determined by analyzing the geometry of the surface of prostate capsule, and the similarity between the boundary landmarks in histological and MR images is calculated by the corresponding geometric features. The internal landmarks are determined by using a scale-space analysis method, which provides the saliency, location, and size of the local blob-like structure. Moreover, the similarity between two internal landmarks respectively in histological and MR images is determined by NMI between the local images around the internal landmarks under comparison. Finally, the correspondences among the automatically detected landmarks and the dense transformation between histological and MR images are simultaneously determined by maximizing an overall similarity function that integrates the similarities between landmarks with spatial constraints.

Experimental results have shown that our proposed method can register anatomical landmarks within prostate capsules at a relatively accurate rate. Also, it can automatically label cancerous regions in MR image by using the cancerous regions reliably detected in histological images, thus facilitating us to learn the profile of cancerous tissues in MR images from a sufficient number of samples in the future. This is 
important for achieving image-based optimal biopsy using patient-specific information, as we mentioned in the beginning of this paper.

\section{References}

1. D. Shen, Z. Lao, J. Zeng, W. Zhang, I. A. Sesterhenn, L. Sun, J. W. Moul, E. H. Herskovits, G. Fichtinger, and C. Davatzikos, "Optimization of Biopsy Strategy by A Statistical Atlas of Prostate Cancer Distribution," Medical Image Analysis, vol. 8, pp. 139150, 2004.

2. Y.Zhan and D. Shen, "Deformable Segmentation of 3-D Ultrasound Prostate Images Using Statistical Texture Matching Method", IEEE Trans. on Medical Imaging, vol. 25, pp. 245-255, March 2006.

3. A. Madabhushi, M. Feldman, D. N. Metaxas, D. Chute, and J. Tomaszewski, "A Novel Stochastic Combination of 3D Texture Features for Automated Segmentation of Prostatic Adenocarcinoma from High Resolution MRI," presented at MICCAI, 2003.

4. I. Chan, W. Wells, 3rd, R. V. Mulkern, S. Haker, J. Zhang, K. H. Zou, S. E. Maier, and C. M. Tempany, "Detection of prostate cancer by integration of line-scan diffusion, T2mapping and T2-weighted magnetic resonance imaging; a multichannel statistical classifier," Med Phys, vol. 30, pp. 2390-8, 2003.

5. L. Taylor, B. Porter, G. Nadasdy, P. di Sant'Agnese, D. Pasternack, Z. Wu, R. Baggs, D. Rubens, and K. Parker, "Three-dimensional registration of prostate images from histological and ultrasound," Ultrasound Med Biol, vol. 30, pp. 161-168, 2004.

6. M. Jacobs, J. Windham, H. Soltanian-Zadeh, D. Peck, and R. Knight, "Registration and warping of magnetic resonance images to histological sections," Medical Physics, vol. 26, pp. 1568-1578, 1999.

7. T. Schormann and K. Zilles, "Three-dimensional linear and nonlinear transformations: An integration of light microscopical and MRI data," Human Brain Mapping, vol. 6, pp. 339347, 1998.

8. A. d. B. d'Aische, M. D. Craene, X. Geets, V. Gregoire, B. Macq, and S. K. Warfield, "Efficient multi-modal dense field non-rigid registration: alignment of histological and section images," Medical Image Analysis, vol. 9, pp. 538-546, 2004.

9. H. Chui and A. Rangarajan, "A new point matching algorithm for non-rigid registration", Computer Vision and Image Understanding, 89:114-141, 2003.

10. D. Shen, E. Herskovits, and C. Davatzikos, "An adaptive focus statistical shape model for segmentation and shape modeling of 3D brain structures," IEEE Trans. on Medical Imaging, vol. 20, pp. 257-271, 2001.

11. T. Lindeberg, "Feature detection with automatic scale selection," International Journal of Computer Vision, vol. 30, pp. 77-116, 1998.

12. F. Maes, A. Collignon, D. Vandermeulen, G. Marchal, and P. Suetens, "Multimodality Image Registration by Maximization of Mutual Information," IEEE Trans. on Medical Imaging, vol. 16, pp. 187-198, 1997.

13. M. Jenkinson and S.M. Smith, "A global optimization method for robust affine registration of brain images", Medical Image Analysis, 5(2):143-156, June 2001.

14. C. Davatzikos, F. Abraham, G. Biros and R. Verma, "Correspondence Detection in Diffusion Tensor Images", ISBI 06', April 2006.

15. A. Andronache, P. Cattin, and G. Székely, "Adaptive Subdivision for Hierarchical Nonrigid Registration of Multi-modal Images Using Mutual Information," presented at MICCAI 2005, 2005. 